\title{
Distinct element modeling of strength variation in jointed rock masses under uniaxial compression
}

\author{
Tao Wang • Dapeng Xu • Derek Elsworth • \\ Weibo Zhou
}

Received: 10 September 2015/Accepted: 7 November 2015/Published online: 19 November 2015

(C) Springer International Publishing Switzerland 2015

\begin{abstract}
Rock masses exhibit strong anisotropy due to the structure of fracture networks embedded within the mass. We use a particulate discrete element method to quantitatively investigate the effect of spacing and inclination angle of the joints on anisotropic strength under uniaxial compression. In all of the numerical models, the intact rock masses are represented by the bonded particle model, and the joint planes are simulated by the smooth-joint model. Observations are made of the evolving stress-strain curves relative to the distribution and orientation of micro fractures. Apparent from this is that: (1) for the same fracture spacing, the strength and deformation parameters of jointed rock masses change in a " $U$ " shaped curve when plotted against an increase in the inclination angle of the joints. The maximum value occurs at the inclination angles of $0^{\circ}$ and $90^{\circ}$, and the minimum value occurs at an inclination angle of $40^{\circ}$ or
\end{abstract}

T. Wang $(\bowtie) \cdot$ D. Xu

State Key Laboratory of Water Resources and

Hydropower Engineering Science, Wuhan University,

Wuhan, China

e-mail: htwang@whu.edu.cn

D. Elsworth

Department of Energy and Mineral Engineering, G3

Center and EMS Energy Institute, Penn State University,

University Park, PA, USA

W. Zhou

Northwest Engineering Corporation Limited, Power

China, Xi'an, China $50^{\circ}$; (2) for the same joint spacing, the failure mode of the jointed rock mass with different joint inclinations can be classified into three categories: splitting tensile failure, shear sliding failure along the joint surface and mixed failure of the two modes above; (3) for the same joint inclination angle, the normalized compressive strength and normalized elastic modulus both increase with an increase of the normalized joint plane layer spacing.

Keywords Uniaxial compression - Discrete element method $\cdot$ Jointed rock masses $\cdot$ Anisotropy

\section{Introduction}

Anisotropy is one of the most distinct features that must be considered in rock engineering because many rocks exposed near the Earth's surface show welldefined fabric in terms of bedding,stratification, layering, foliation, fissuring, or jointing (e.g., Everitt and Lajtai 2004; Duan and Kwok 2015). The mechanical properties of a jointed rock mass are complex because of its high textural anisotropy. The strength and deformability of a jointed rock mass are heavily influenced by the orientation and the distribution of the joints, and the failure mode varies significantly with the variation of the joint orientation (Hoek 2007).

Therefore, the mechanical behavior of a jointed rock mass is an important topic in rock engineering. 
Numerous previous studies have investigated the mechanical properties of a jointed rock mass through uniaxial or triaxial compression tests (Yang et al. 1998). The uniaxial compression strength (UCS) and Young's modulus of the Danba schist at different dip angles were studied via uniaxial compression tests (Chu et al. 2013). The influence of joint orientation on the fracture progression behavior of singly-jointed sandstone under undrained triaxial conditions is researched (Wasantha et al. 2015). The experimental investigation of deformation and strength anisotropy of Asan gneiss, Boryeong shale and Yeoncheon schist in Korea give clear evidence of transverse isotropy (Cho et al. 2012). Moreover, to systematically investigate the influence of the joint orientation, artificial rock specimens have often been used to mimic the response of natural rock (Kulatilake et al. 2001). In addition to these experiments, Jaeger (1972) employed stress superposition theory to predict the variation in strength of a jointed rock mass with one or two sets of joints. Various advanced models combined with either empirical or theoretical formulae have been proposed to explore the more complex phenomena of a rock mass, such as the failure mode and the sliding behavior between blocks. A method that incorporates the deformation and the strength of intact rock and joints is proposed such that the complete pre- and post-peak deformation of rock mass can be obtained (Wang and Huang 2009). Considering the unique stratigraphic characteristics of salt rocks, a new Cosserat-like medium constitutive model is proposed to facilitate the efficient modeling of the mechanical behavior of these formations ( $\mathrm{Li}$ et al. 2009). These models could be further incorporated within continuum numerical analysis techniques based on the concept of an equivalent continuum. The equivalent continuum treats the rock mass as a homogeneous anisotropic body, which superposes the intact rock properties and the joint behavior at different orientations, to describe the overall mechanical behavior of the rock mass. Thus, using this concept, continuum numerical analysis can follow complex rock mass behavior.

However, jointed rock masses are discontinuous and anisotropic, in which the fractures in the intact rock interact with the sliding of an existing joint face. To consider the sliding effect, certain studies have used discrete element methods (DEM) to define failure processes and resulting strength of the rock mass. A PFC numerical model was able to reproduce the damage zone observed in laboratory experiments (Fakhimi et al. 2002). Investigation of the failure evolution of simulated Lac du Bonnet granite has also been performed, in which the number and type of contact failures (micro cracks) are monitored (Wang and Tonon 2009). To explore the effects of jointing on strength, the fracture system is linked to PFC models. Simulated Rock Mass (SRM) models containing thousands of non-persistent joints can be virtually fabricated and correspondence to standard laboratory tests (UCS, triaxial loading, and direct tension tests) or tested under a non-trivial stress paths used to represent the behavior of the mass (Mas Ivars et al. 2011). A new clumped-particle logic set of micro parameters was used to predict the strength of the synthetic rock, independent of the stress path (Cho et al. 2007).

In this study, a numerical technique combing a bonded particle model with a smooth-joint model (SJM) is proposed to represent the weak layers in inherently anisotropic rocks. The influence of the dip angle and spacing on strength and deformation of the jointed rock mass is examined in detail. The numerical results are quantitatively compared with previous experimental and analytical results. The failure mode and fracture direction are investigated.

\section{Numerical models}

Particle-flow code (PFC) models the movement and interaction of circular particles using the DEM, as described by Cundall and Strack (1979). PFC has three advantages. First, PFC is potentially more efficient because contact detection between circular objects is much simpler than contact detection between angular objects; second, there is essentially no limit to the extent of displacement that can be modeled; and third, it is possible for the blocks to break (because they are composed of bonded particles). The constitutive behavior of a material is simulated in PFC by associating a contact model with each contact. In addition to the contact model, there may be a bond model and a dashpot. These three components, in their entirety, define the contact force-displacement behavior (Itasca 2010).

\subsection{Bonded model}

Two basic bond models are provided in PFC: a "Contact Bond (CB) model" and a "Parallel Bond 
Fig. 1 Numerical model used for modeling rock joints

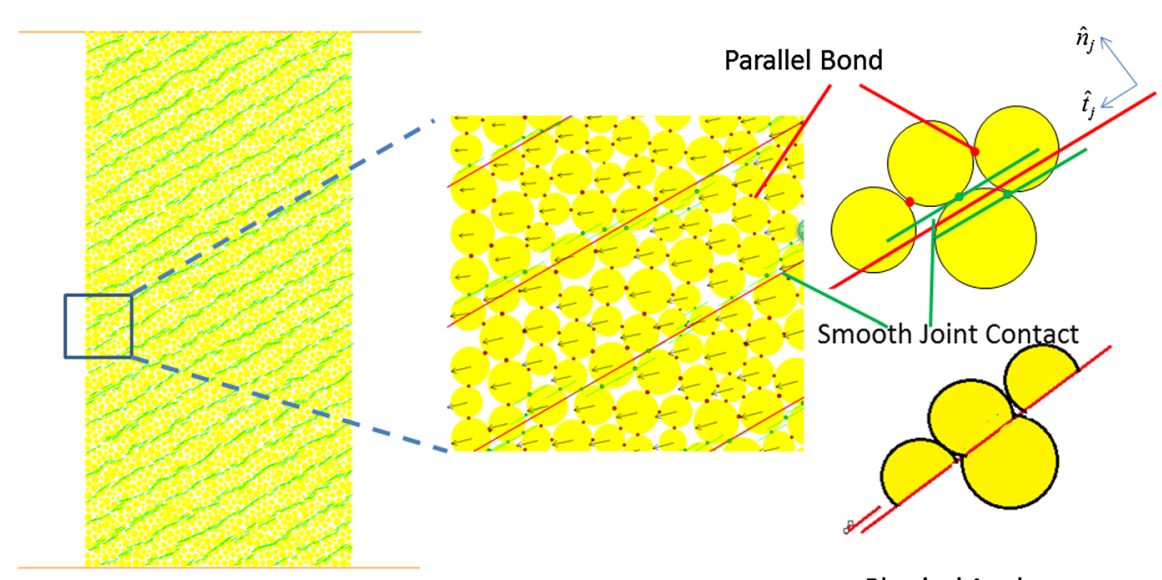

Physical Analogue
(PB) model". A CB model can be envisaged as a pair of elastic springs with a constant normal and shear stiffness acting at a point. A PB model approximates the physical behavior of a cement-like substance joining the two particles. The PB model establishes an elastic interaction between particles that act in parallel with the slip or contact-bond constitutive models. Particles in the PFC are free to move in the normal and shear direction and can rotate between particles. This rotation may induce a moment between particles, but the CB model cannot resist this moment. With the PB model, however, bonding is activated over a finite area; thus, this bonding can resist a moment.

\subsection{Contact model}

By default, all contacts are assigned either the linear or Hertz model, depending on the properties of their two contacting entities (ball-ball or ball-wall). The linear model includes contact-bond behavior (i.e., the linear model may be bonded or unbonded, and when bonded, it behaves as a contact bond). Parallel-bond behavior is implemented by adding a parallel-bond component to a contact, and this component acts in parallel with the other components at the contact. Before describing the linear and Hertz models, we describe the component behaviors that are provided by these models.

The SJM was first proposed to model the presence of joints in fractured rock masses. The SJM simulates the behavior of an interface, regardless of the local particle contact orientation along the interface. The behavior of a joint can be modeled by assigning smooth joint models to all contacts between particles that lie on the opposite side of the joint. The joint can be envisioned as a set of elastic springs uniformly distributed over a rectangular cross-section, centered at the contact point and oriented parallel with the joint plane. A typical smooth-joint contact in 2D is illustrated in Fig. 1.

The SJM provides the macroscopic behavior of a linear elastic and either bonded or frictional interface with dilation. The behavior of the bonded interface is linear elastic until the strength limit is exceeded, at which point the bond breaks, which causes the interface to become unbonded. The behavior of an unbonded interface is linear elastic and frictional with dilation, with slip accommodated by imposing a Coulomb limit on the shear force. The interface does not resist relative rotation.

When the SJM is created, the parallel bond will be deleted and replaced by the smooth joint contact model. Particles intersected by a smooth-joint can pass through each other by sliding along the pre-defined joint surface rather than moving around one another. Because the smooth-joint orientation is not aligned with the contact normal, the relative displacement increment is resolved into joint normal and shear directions:

$\Delta \delta=\Delta \widehat{\delta}_{n} \hat{\mathbf{n}}_{j}+\Delta \widehat{\delta}_{s}$

The accumulated normal and shear displacements are updated as

$\widehat{\delta}_{n}=\widehat{\delta}_{n}-\Delta \widehat{\delta}_{n}$
$\widehat{\delta}_{s}=\widehat{\delta}_{s}+\Delta \widehat{\delta}_{s}$

The elastic portions of the normal $\Delta \hat{\delta}_{n}^{e}$ and shear displacement increments $\Delta \widehat{\delta}_{s}^{e}$ are determined based on 
the value of the gap: if they are bonded, then the entire displacement increment is elastic; if they are not bonded, then only the portion of the displacement increment that occurs is elastic.

The force-displacement law for the SJM updates the contact force:

$\mathbf{F}_{c}=\mathbf{F}, \mathbf{M}_{c} \equiv 0$

where is the smooth-joint force. The force is resolved into normal and shear forces:

$\mathbf{F}=-F_{n} \hat{\mathbf{n}}_{j}+\mathbf{F}_{s}$

The SJM has been successfully applied to the study of fractured rock mass failure (Mas Ivars et al. 2011), the scale effect (Zhang et al. 2011), anisotropic behavior of jointed rock masses (Chiu et al. 2013) and transversely isotropic rocks (Park and Min 2015).

\section{Simulation of uniaxial compression of jointed rock masses}

The uniaxial or unconfined compressive strength (UCS) of rock is an important and widely used parameter in rock mechanics and rock engineering. To determine the UCS, laboratory, in situ and numerical compression tests are usually conducted. The PFC uniaxial compression numerical test is shown in Fig. 2.

\subsection{Generation of jointed rock masses model}

The dimensions of the numerical model sample $(\mathrm{W} \times \mathrm{H}=0.05 \mathrm{~m} \times 0.1 \mathrm{~m})$ are selected according

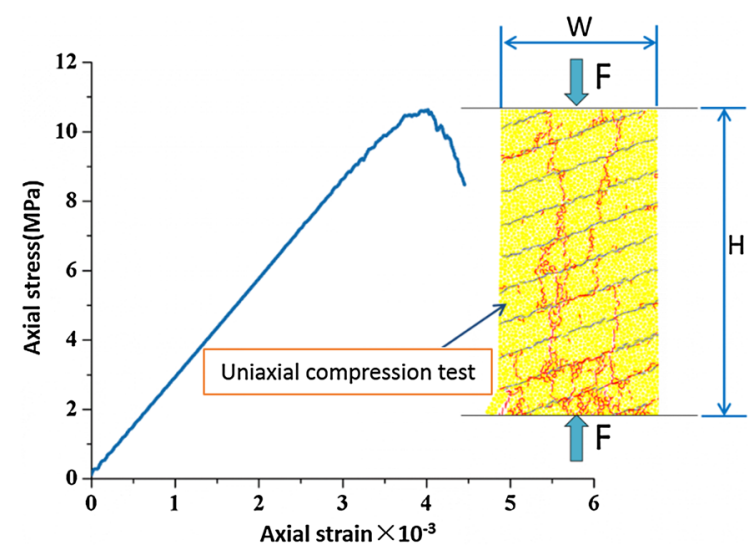

Fig. 2 Uniaxial compression numerical test
Table 1 Geometrical parameters of uniaxial compression test sample

\begin{tabular}{ll}
\hline Geometrical parameters & Value \\
\hline Sample size $(\mathrm{m})$ & $\mathrm{W} \times \mathrm{H}=0.05 \times 0.1$ \\
Minimum particle size $(\mathrm{m})$ & $\mathrm{R}_{\min }=0.0005$ \\
Particle size ratio & $\mathrm{r}=1.66$ \\
Porosity & $\mathrm{n}=0.15$ \\
Particle number & $\mathrm{N}=3311$ \\
Particle density $\left(\mathrm{kg} / \mathrm{m}^{3}\right)$ & $\rho_{\text {partical }}=2900$ \\
\hline
\end{tabular}

Where $\rho_{\text {partical }}=\frac{\rho}{1-n}$ and $\rho$ is the macro density of the material

to the conventional indoor physical and mechanical tests, the relevant mechanical properties of rock masses and the method of determination. The particle assembly in the sample was generated using the radius expansion method. The particle size follows a uniform distribution, with $\mathrm{Rmin}=0.5 \mathrm{~mm}$ and Rmax/ Rmin $=1.66$, as listed in Table 1 . The generation of a layered rock sample consists of two main steps: first, performing particle assembly specified by using the bonded particle model (BPM) contact model and the corresponding micro-mechanical parameters to simulate the intact rock blocks; second, adding the weak layers, which are specified using the SJM contact model, and inputting the related micro-mechanical parameters into the intact rock model.

\subsection{Simulation procedure for uniaxial compression numerical test}

The PFC modeling process can be divided into the following 4 steps.

1. Compact initial assembly of the particles

The compression tests are performed in a polyaxial cell. The top and bottom walls act as loading platens, and the velocities of the sidewalls are controlled by a servomechanism to maintain a constant confining stress. All of the side walls are removed for fully unconfined tests, which are performed by setting the stress $\psi$ equal to a nonzero value. All walls are frictionless, and the normal stiffness of the platen walls and the confining walls are set equal to $\psi$ the average particle normal stiffness of the material. The particle diameters satisfy a certain particle-size distribution and are bounded by $D_{\min }$ and $D_{\max }$. 
2. Apply an initial confining stress

The compression test begins with a seating phase. Axial and confining stresses are applied by activating the servomechanism for all opposing $\psi$ walls and then cycling under control of the solver until either the stresses are applied successfully or it is determined that $\psi$ the target stresses cannot be reached. The servomechanism can be activated independently for each set of the opposing $\psi$ walls. The servo behavior is controlled by the wall-servo tolerance such $\psi$ that the corresponding velocity will be zero when the value is reached. After the axial and confining stresses have been applied to the specimen, the specimen dimensions at this stage are taken as the initial dimensions to be used in the computation of stresses and strains during the subsequent loading phase.

\section{Apply constant strain rate}

The specimen is loaded by moving the platens toward one another at a final velocity, which is determined by specifying the strain rate. The platen acceleration at the start of loading is controlled by specifying the appropriate values. The platen velocity will be adjusted to reach a final value in a sequence of stages. Note that a value of strain rate that $\psi$ is sufficient to produce quasi-static loading on a specimen of initial length value will most likely not produce quasi-static loading on a specimen of a different length.

\section{Loading process}

The loading phase continues until the specified testtermination criterion is reached. The deviatoric stress is monitored, and the maximum value is recorded. During a typical test on a bonded material, this value of the deviatoric stress will increase to some maximum and then decrease as the specimen fails, and the test can be terminated when the deviatoric stress is less than a tolerance value.

\section{Effect of joints on the mechanical properties of rock masses}

\subsection{Effect of joint inclination}

\subsubsection{Numerical test schemes}

The uniaxial compression numerical tests are based on the shale (selected from a project), whose parameters
Table 2 Mesoscopic parameters of the BPM and the SJM

\begin{tabular}{llll}
\hline $\mathrm{BPM}$ & \multicolumn{3}{l}{$\mathrm{SJM}$} \\
\hline$R_{\min } / \mathrm{m}$ & 0.05 & $\bar{k}_{n} / \mathrm{GPa} / \mathrm{m}$ & 4000 \\
$R_{\max } / R_{\min }$ & 1.66 & $\bar{k}_{s} / \mathrm{GPa} / \mathrm{m}$ & 500 \\
$\rho / \mathrm{kg} / \mathrm{m}^{3}$ & 2600 & $\bar{\lambda}$ & 1.0 \\
$M$ & 0.7 & $M$ & 0.8 \\
$E_{c} / \mathrm{GPa}$ & 15.0 & $\psi /^{\circ}$ & 0 \\
$k_{n} / k_{s}$ & 4.0 & $M$ & 3 \\
$\bar{\lambda}_{p B}$ & 1.0 & $\sigma_{c} / \mathrm{MPa}$ & 12.0 \\
$\bar{E}_{c} / \mathrm{GPa}$ & 10.0 & $c_{b} / \mathrm{MPa}$ & 6.0 \\
$\bar{\sigma}_{c} / \mathrm{MPa}$ & 28 & $\varphi_{b}{ }^{\circ}$ & 0 \\
$\bar{\tau}_{c} / \mathrm{MPa}$ & 28 & & \\
$\bar{k}^{n} / \bar{k}^{s}$ & 4.0 & & \\
\hline
\end{tabular}

Table 3 Numerical test scheme and results of rock masses with different joint inclination angles

\begin{tabular}{lcll}
\hline Sample number & Inclination angle $/ \theta^{\circ}$ & $\bar{E}$ & $\bar{\sigma}$ \\
\hline $1^{\#}$ & 0 & 0.86 & 0.96 \\
$2^{\#}$ & 10 & 0.82 & 0.95 \\
$3^{\#}$ & 20 & 0.77 & 0.94 \\
$4^{\#}$ & 30 & 0.70 & 0.88 \\
$5^{\#}$ & 40 & 0.67 & 0.47 \\
$6^{\#}$ & 50 & 0.67 & 0.38 \\
$7^{\#}$ & 60 & 0.71 & 0.38 \\
$8^{\#}$ & 70 & 0.79 & 0.49 \\
$9^{\#}$ & 80 & 0.86 & 0.72 \\
$10^{\#}$ & 90 & 0.90 & 0.91 \\
\hline
\end{tabular}

are shown in Table 2, and the calculation scheme is shown in Table 3. The inclination angle is "positive" when the joint plane rotates counterclockwise from the horizontal plane, changing uniformly from $0^{\circ}$ to $90^{\circ}$ at a $10^{\circ}$ interval. A total of 10 rock samples were tested, with all of joint plane spacing taken as $0.01 \mathrm{~m}$ and all of the joints distributed throughout the model. The rock samples of inclination angles of $0^{\circ}, 30^{\circ}, 60^{\circ}$ and $90^{\circ}$ are shown in Fig. 3.

\subsubsection{Stress-strain curve}

Uniaxial compression numerical tests of the various rock samples were performed in accordance with the procedure described in Sect. 3.2; the axial stressstrain curves of the samples are shown in Fig. 4. The stress-strain curves of rock samples with different 
Fig. 3 Rock samples of different joint inclination angles
Fig. 4 Stress-strain curves of the rock samples with different inclination angles

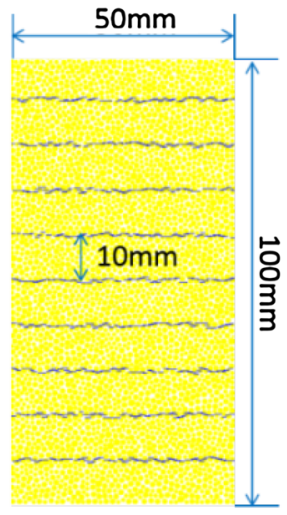

$\operatorname{dip}=0^{\circ}$

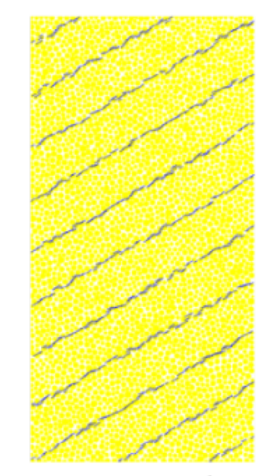

$\operatorname{dip}=30^{\circ}$
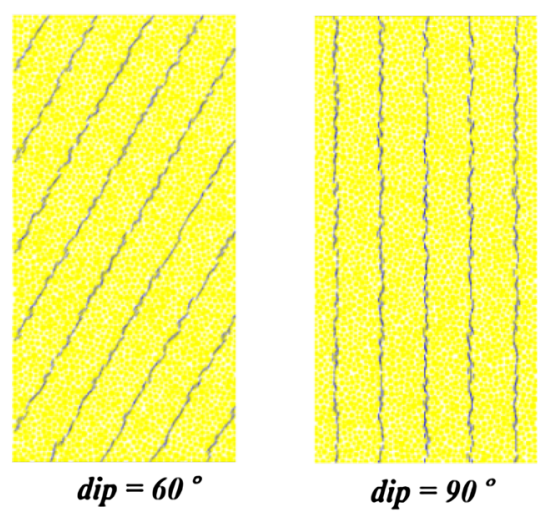

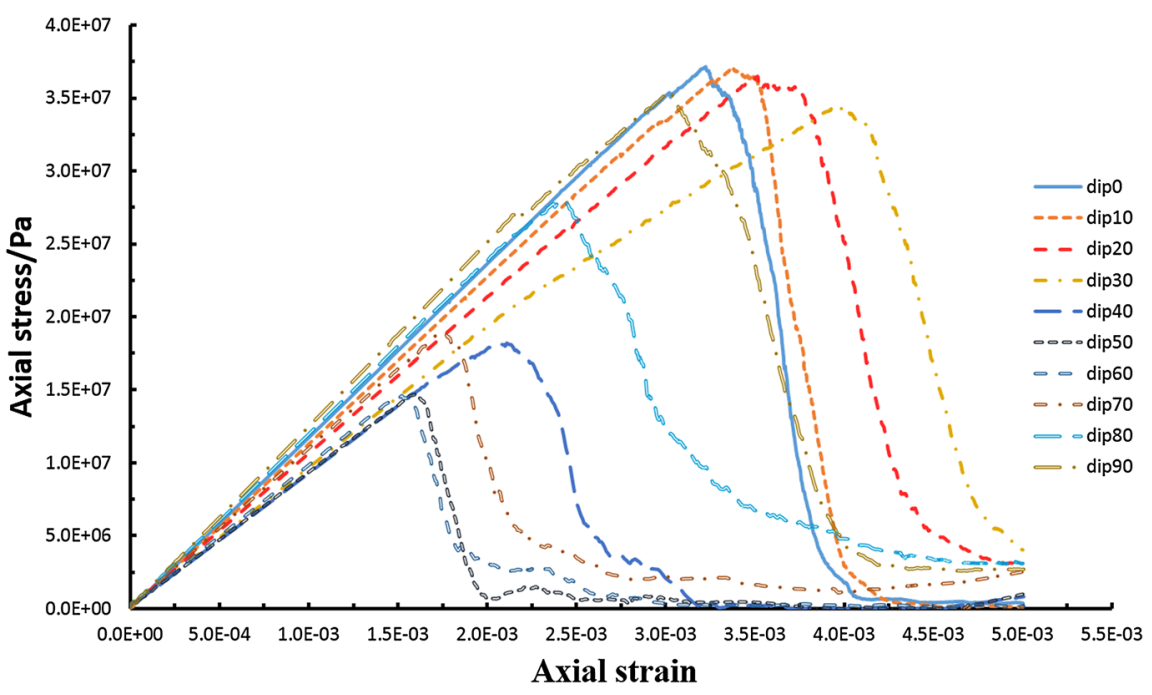

inclination angles show clear differences, and the elastic modulus and compressive strength of the samples both initially decrease and then increase with the increase of the inclination angle from $0^{\circ}$ to $90^{\circ}$. After the peak axial stress, all of the axial stress curves decrease rapidly with nearly the same slope, which illustrates that under the same joint spacing of $0.01 \mathrm{~m}$, the rock samples with different joint inclination angles exhibit the similar brittle nature.

\subsubsection{Crack distributions}

\section{Propagation of micro cracks}

The crack distributions of rock samples with different joint inclination angles are shown in Fig. 5, and the failure mode of the rock samples can be classified into three categories:

Splitting-mode splitting tensile failure along the longitudinal direction (e.g., at $0^{\circ}$ and $90^{\circ}$ ), which can be confirmed from the distribution of micro-cracks in the rock samples.

Sliding-mode shear sliding failure along the joint planes (e.g., at $40^{\circ}$ and $70^{\circ}$ ). Because of the low bond strength of the joints, shear sliding failure tends to occur along the joint planes in the rock masses and is accompanied by the generation of micro-cracks near the joint planes.

Mixed-mode mixed failure of the two modes above (e.g., at $20^{\circ}$ and $30^{\circ}$ ). The breakage occurs in intact rock, and stepped failure is generated through the local adjacent joint planes. 


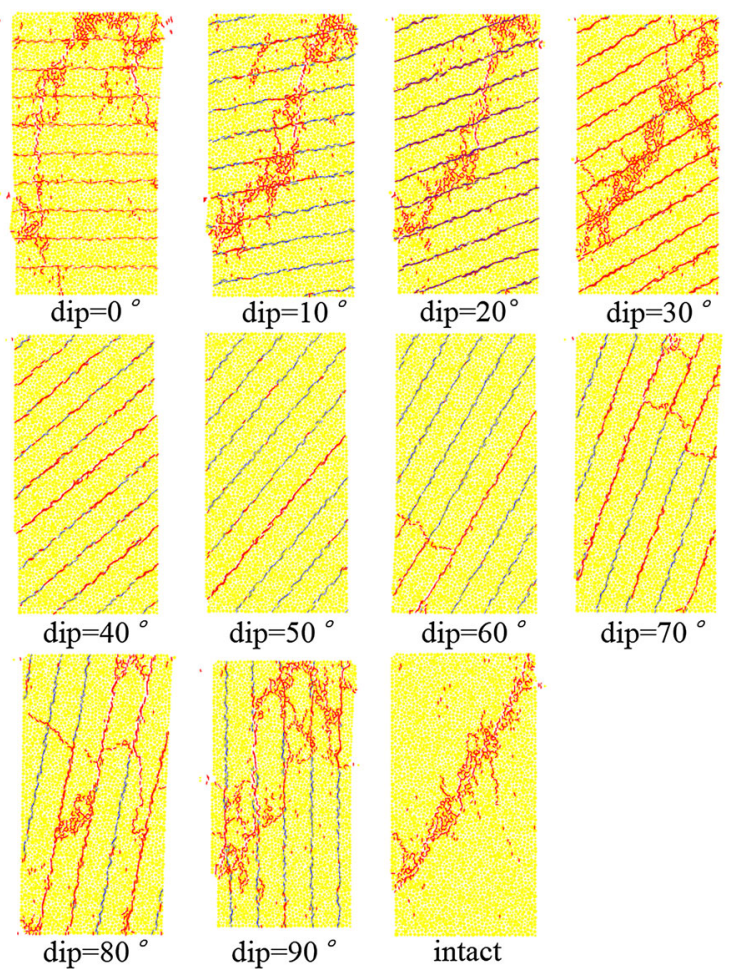

Fig. 5 Crack distributions of rock samples with different joint inclination angles with the axial strain rate in each sample model can be divided into three stages. In Stage 1, micro-cracks rarely occur at the initial stage before the axial strain reaches $0.15 \%$ (note that it was $0.2 \%$ for the uniaxial compression test) (Duan and Kwok 2015); in Stage 2, first, the number of micro-cracks increases slowly to a certain value and then increases rapidly until the model is destroyed; and in Stage 3, the number of micro-cracks tends to be stable and no longer changes.

As shown in Fig. 7, different stages of the microcrack distribution occur during the uniaxial compression test process. During the test, the breaking of smooth joint contacts first induces micro-cracks, and then the parallel bonds break to generate micro-cracks sequentially; as a result, there are two periods of rapid growth in the second stage in the curve.

\section{Orientation of the micro-cracks}

Figure 8 illustrates the rose diagrams of the inclination angle of the micro-cracks in the ultimate failure state. The inclination angle of a micro-crack is "positive" when the crack rotates counterclockwise from the horizontal plane, which is consistent with the "positive" direction of the joint plane. To simplify the statistics, the following equation is used in the process of statistical processing of the crack inclination angle:

$\theta_{s}=\left\{\begin{array}{cl}(10 m)^{\circ} & \left.(10 m-5)^{\circ} \leq \theta<(10 m+5)\right)^{\circ},-8 \leq m \leq 8, m \in Z \\ -90^{\circ} & -90^{\circ} \leq \theta<-85^{\circ} \\ 90^{\circ} & 85^{\circ} \leq \theta \leq 90^{\circ}\end{array}\right.$

Therefore, when the inclination angle is between $40^{\circ}$ and $70^{\circ}$, the damage area distribution of the sample is easily affected by joints, whereas the joints have little effect on the damage area distribution when the joint plane is nearly parallel or vertical to the axial stress direction.

Under different joint inclination angles, the curves of the cumulative crack number-axial strain rate in rock samples under uniaxial numerical compression are shown in Fig. 6. The cumulative crack number is the total number of microcracks induced by the loading-and defined as particle-particle detachments. The development of cumulative crack number where $\theta$ is the real inclination angle and $\theta_{s}$ is the statistical inclination angle. As shown in Fig. 8, the radial length of each bin indicates the number of micro-cracks oriented within the angles defined by the bin. The cracks are found to have a preferential vertical orientation, which is parallel to the direction of the joint plane. This preference is mainly due to the low bond strength of the joints, which are easy to break under the action of axial stress and lead to the formation of micro-cracks. The micro-cracks extend along the joint plane and eventually run through the entire sample model, thus leading to the failure of the model. 
Fig. 6 Cumulative crack number-axial strain rate for different joint inclination angles

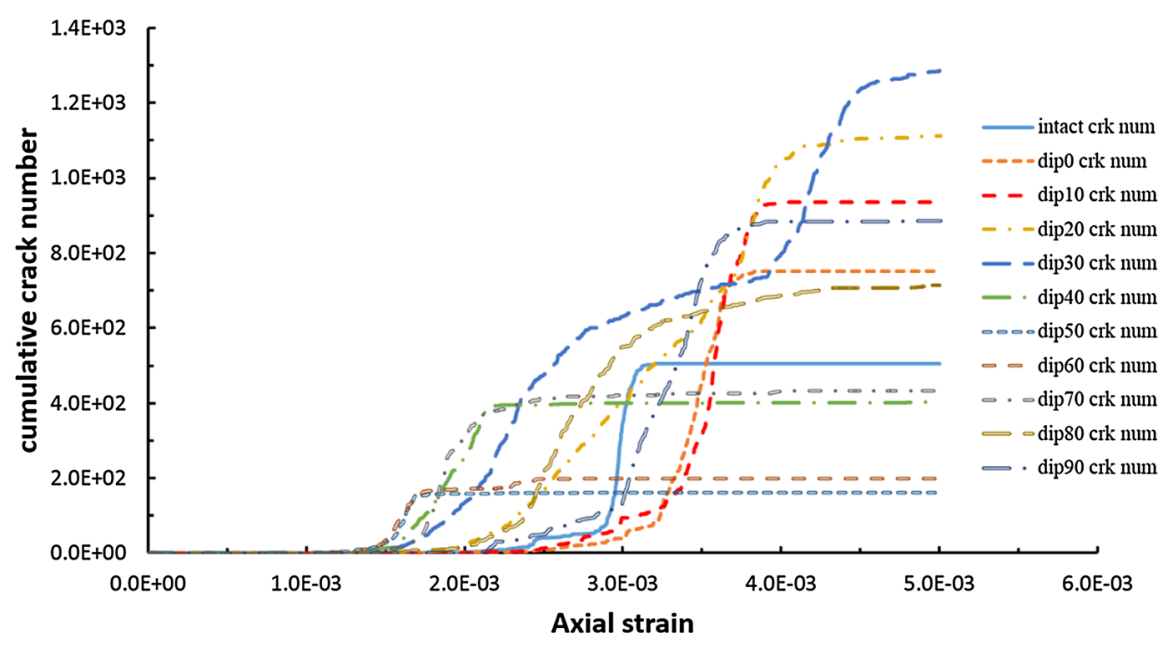

Fig. 7 Micro-crack distribution at different stages $\left(30^{\circ}\right)$
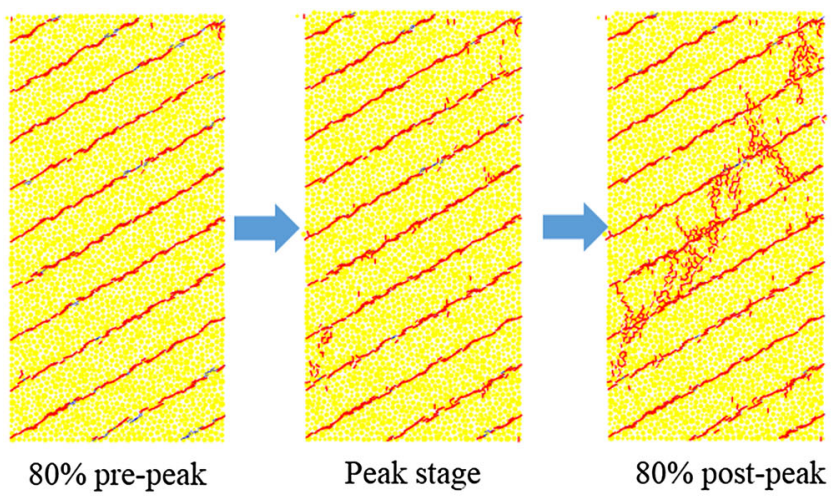

Broken stage(axial strain $=0.5 \%$ )

\subsubsection{Simulation results of the elastic modulus and the compressive strength}

According to the curves in Fig. 4, the uniaxial elastic modulus and the compressive strength of each rock sample can be calculated. The normalized compressive strength $\bar{\sigma}$ and normalized elastic modulus $\bar{E}$ can be obtained as follows:

$$
\begin{gathered}
\bar{\sigma}=\frac{\sigma_{j R}}{\sigma_{i R}} \\
\bar{E}=\frac{E_{j R}}{E_{i R}}
\end{gathered}
$$

where $\sigma_{j R}$ and $\sigma_{i R}$ are the uniaxial compressive strength of jointed rock masses and intact rock masses, respectively, and the elastic modulus of jointed rock masses and intact rock masses are $E_{j R}$ and $E_{i R}$, respectively. The value of the normalized elastic modulus and the compressive strength are less than 1, which reflects the weakening influence of the joints on the strength and deformation properties of the rock masses.

The values of the normalized elastic modulus and compressive strength calculated according to Eqs. 7 and 8 , respectively, are listed in Table 3 . The curves of the normalized elastic modulus and the compressive strength versus the inclination angle are shown in Figs. 9 and 10, respectively. The analytical solution of the elastic modulus with an inclination angle of $\theta\left(E_{\theta}\right)$ can be calculated by (Amadei 1982):

$\frac{1}{E_{\theta}}=\frac{1}{E_{i R}}+\cos ^{2} \theta\left(\frac{\cos ^{2} \theta}{\bar{k}_{n} \delta}+\frac{\sin ^{2} \theta}{\bar{k}_{s} \delta}\right)$

where $E_{i R}$ is the elastic modulus of intact rock masses, $\bar{k}_{n}$ and $\bar{k}_{s}$ respectively are the normal stiffness and 
Fig. 8 Distribution of orientations of the microcracks
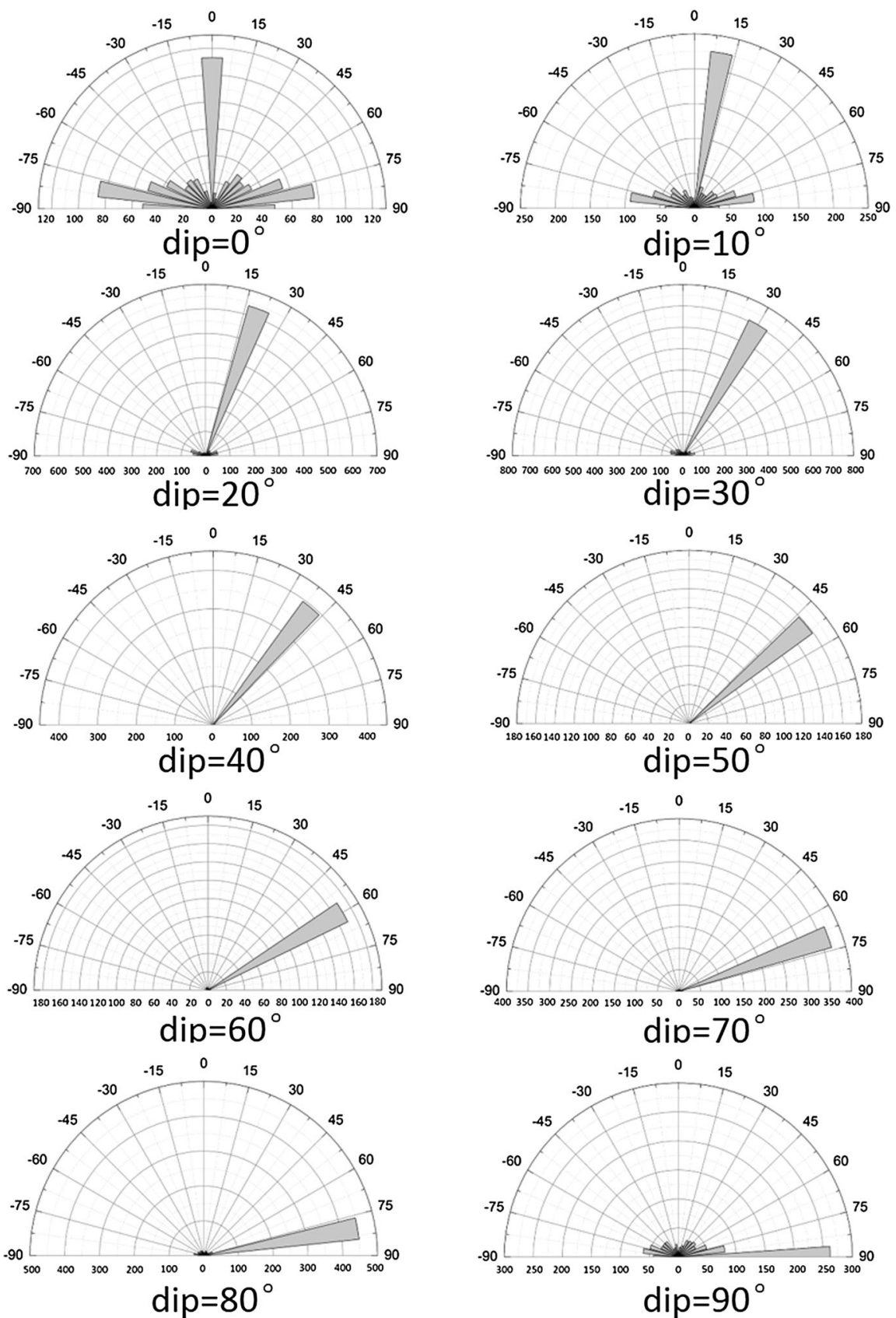

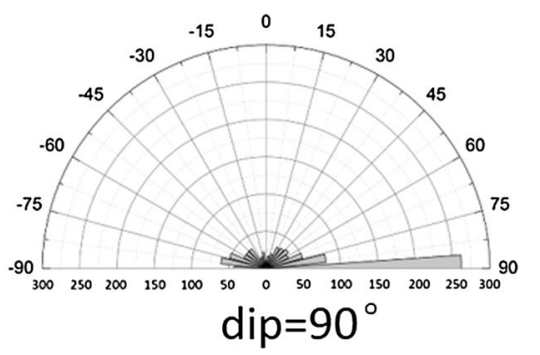

shear stiffness of joint plane, respectively, and $\delta$ is the joint spacing. The uniaxial compressive strength of rock masses can be expressed as a function of friction angle $\left(\phi_{w}\right)$, cohesion $(c)$ and the inclination angle $(\theta)$ of the joint plane, as shown below (Jaeger et al. 2007):

$\sigma_{\theta}=\frac{2 c}{\left(1-\tan \phi_{w} \cot \theta\right) \sin 2 \theta}$
As shown in Figs. 9 and 10, the normalized elastic modulus and compressive strength both change in a " $U$ " type curve with an increase of the inclination angle of the joints. When the inclination angle is between $40^{\circ}$ and $70^{\circ}$, the shear stress along the joint plane is so much larger that the sample easily undergoes shearing slip failure along the joint plane 


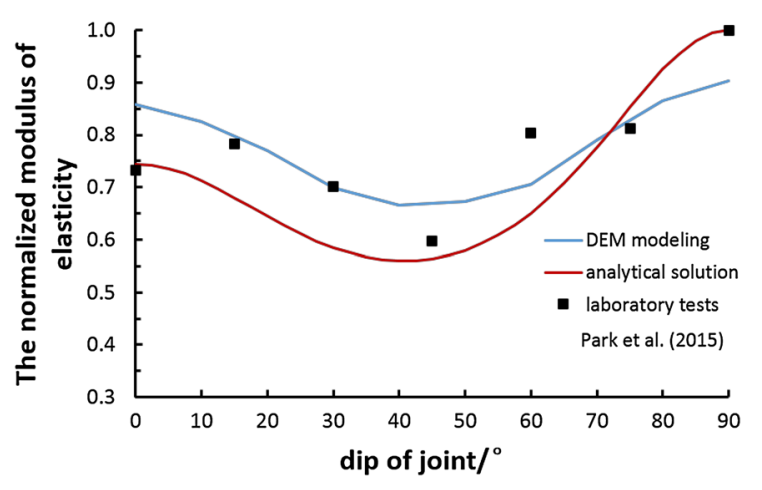

Fig. 9 Variation of the normalized elastic modulus with the joint inclination angle

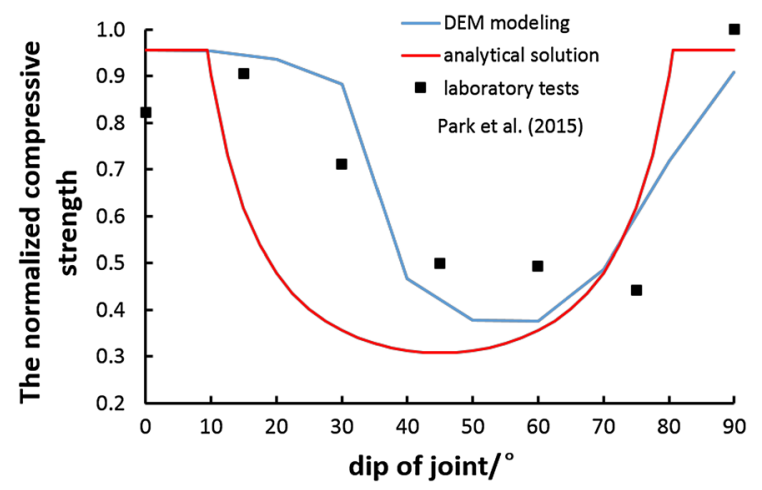

Fig. 10 Variation of the normalized compressive strength with the joint inclination angle

under the action of axial stress; thus, both the elastic modulus and compressive strength reach the minimum accordingly. In contrast, when the inclination angle is $0^{\circ}$ or $90^{\circ}$, the micro cracks are mainly caused by the breaking of parallel bonds in the rock block; therefore, the strength and deformation properties of the rock masses are weakened slightly by the joints.

As observed from Table 3, the normalized elastic modulus decreases from 0.86 to the minimum value of 0.67 with the inclination angle increasing from $0^{\circ}$ to $40^{\circ}$ and then increases with the increase of inclination angle. With the increase in the inclination angle, the normalized compressive strength decreases first and then increases, with the minimum value reached at $50^{\circ}$. Both the normalized elastic modulus and the compressive strength reach the maximum when the angle is $0^{\circ}$ or $90^{\circ}$, which indicates that the weakening degree of the horizontal or vertical joints on the strength and deformation properties of rock masses is the smallest. The ratios of the maximum and minimum values of the elastic modulus and compressive strength are 1.34 and 2.53, respectively. This result shows that the anisotropic influence of joint inclination angle on the strength and deformation of rock masses is significant.

\subsection{Effect of joint spacing \\ 4.2.1 Numerical test schemes}

The joint spacing in rock masses reflects the intensity of structural deformation and is another factor that affects the engineering rock deformation and strength. The computational solution for uniaxial compression of the jointed rock mass is shown in Table 4, where the joint spacing varies uniformly from 0.005 to $0.025 \mathrm{~m}$, with an interval every $0.005 \mathrm{~m}$. For three different failure modes, three types of joint inclinations are selected: $30^{\circ}$ (mixed mode), $60^{\circ}$ (sliding mode), and $90^{\circ}$ (split mode); each model has five samples.

\subsubsection{Stress-strain curve}

As shown in Fig. 11, the stress-strain curve trends are consistent: initially, linear growth occurs, which is followed by a sharp decline after the curve reaches a peak; the rock mass does not undergo rapid brittle failure until the stress value reduces to the residual

Table 4 The number of the numerical test and the result under different joint spacings

\begin{tabular}{lllll}
\hline Sample number & Inclination angle $/ \theta^{\circ}$ & $\bar{S}$ & $\bar{\sigma}$ & $\bar{E}$ \\
\hline $1^{\#}$ & 30 & 0.20 & 0.77 & 0.54 \\
$2^{\#}$ & 30 & 0.40 & 0.88 & 0.70 \\
$3^{\#}$ & 30 & 0.60 & 0.96 & 0.78 \\
$4^{\#}$ & 30 & 0.80 & 0.92 & 0.82 \\
$5^{\#}$ & 30 & 1.00 & 0.98 & 0.86 \\
$6^{\#}$ & 60 & 0.20 & 0.37 & 0.55 \\
$7^{\#}$ & 60 & 0.40 & 0.38 & 0.71 \\
$8^{\#}$ & 60 & 0.60 & 0.40 & 0.79 \\
$9^{\#}$ & 60 & 0.80 & 0.44 & 0.82 \\
$10^{\#}$ & 60 & 1.00 & 0.43 & 0.86 \\
$11^{\#}$ & 90 & 0.20 & 0.73 & 0.82 \\
$12^{\#}$ & 90 & 0.40 & 0.91 & 0.90 \\
$13^{\#}$ & 90 & 0.60 & 0.98 & 0.94 \\
$14^{\#}$ & 90 & 0.80 & 0.93 & 0.94 \\
$15^{\#}$ & 90 & 1.00 & 0.99 & 0.98 \\
\hline
\end{tabular}



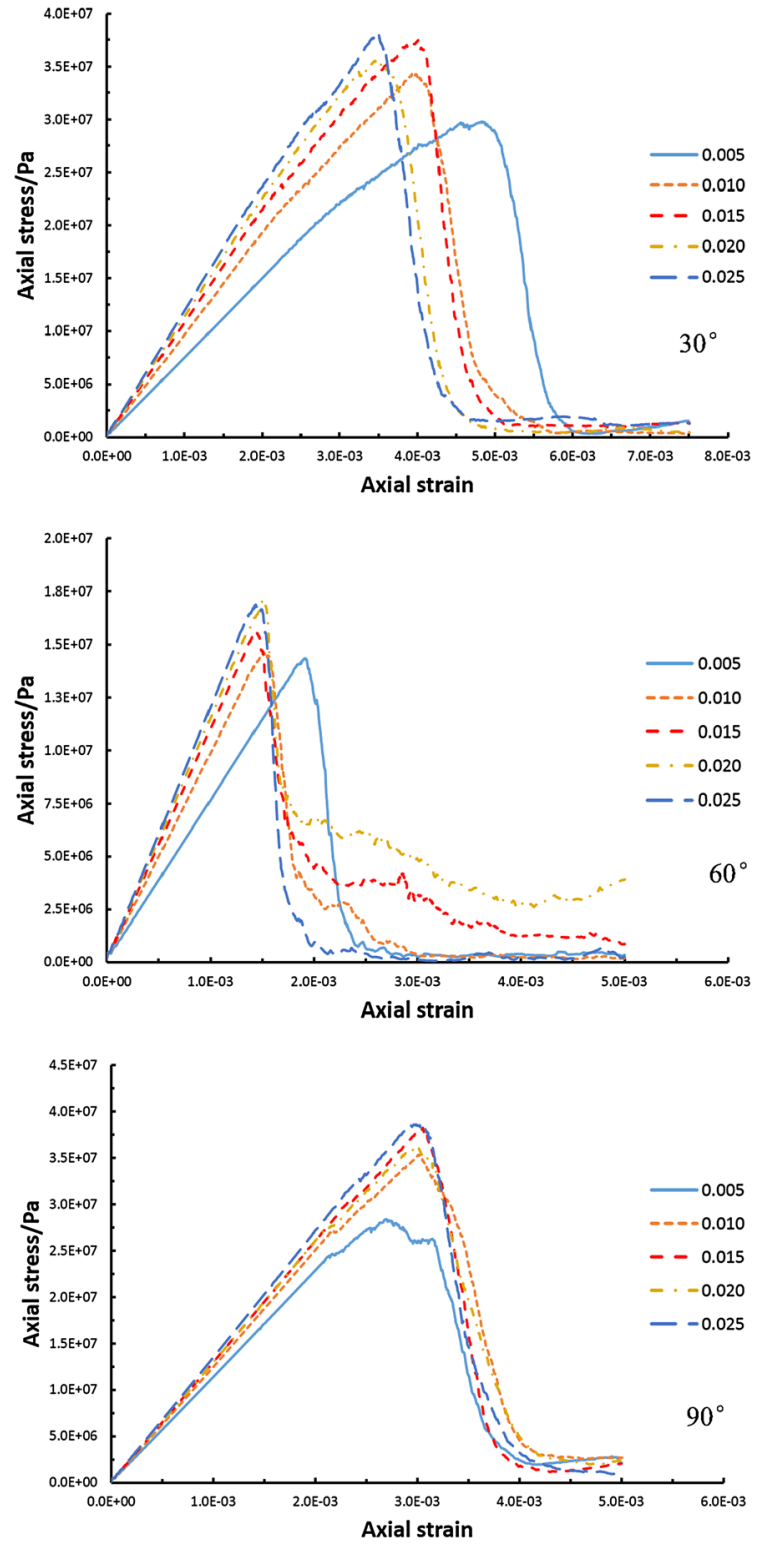

Fig. 11 Stress-strain curves of rock samples with different joint spacings

strength. Among the three groups of samples $\left(30^{\circ}, 60^{\circ}\right.$ and $90^{\circ}$ ), both the compressive strength and the elastic modulus increase with an increase in joint spacing, but the growth rates of the compressive strength and the elastic modulus are different.

\subsubsection{Distribution of cracks}

Under different joint spacings, when the rock mass samples are broken, the propagation of micro-cracks is shown in Fig. 12, 13 and 14. The failure modes of samples are not observed to change with an increase of joint spacing. Thus, the joint inclination has a significant influence on the propagation modes of micro-cracks, but the different joint spacings have little influence.

\subsubsection{Elastic modulus and the compressive strength}

According to the stress-strain curve, the compressive strength and elastic modulus of the rock mass samples can be calculated, and then the compressive strength, elastic modulus and joint spacing may be normalized as follows:

$\bar{S}=\frac{S_{i}}{\max \left(S_{i}\right)}$

where $S_{i}$ is the $i$-th joint spacing, and according to Eqs. 2 and 4, the normalized compressive strength and normalized elastic modulus can be calculated for different joint spacings-for joint inclinations for $30^{\circ}$, $60^{\circ}$, and $90^{\circ}$, as shown in Table 4 . The normalized compressive strength may be evaluated against spacing $\bar{s}$ (Fig. 15) and likewise the normalized elastic modulus (Fig. 16).

According to Figs. 15 and 16, the change in the normalized compressive strength and the normalized elastic modulus versus joint spacing follow similar trends, i.e., they increase slightly with joint spacing. Figure 15 clearly shows that the compressive strength changes with the joint spacing for joint inclinations of $30^{\circ}$ and $90^{\circ}$, but not $60^{\circ}$. Mainly because the intact rock fails in tensile failure in the case of the joint inclination of $30^{\circ}$ and $90^{\circ}$, the smaller joint spacing means that more weak planes occur along the lateral direction and that more joints are therefore opened under load. The joint spacing has a clear effect on the compressive strength in this situation. However, the rock mass mainly undergoes shear slip failure along several joint planes in the case of the joint inclination at $60^{\circ}$, and the change of the joint spacing does not make the number of shear slip failure plane increase significantly. Thus the joint spacing has only a small effect on the compressive strength in this case. From Fig. 16, it can be observed that the variation of elastic modulus with joint spacing is relatively similar to the case of rock mass strength.

Both the uniaxial compressive strength and the normalized elastic modulus with the normalized joint 
Fig. 12 Distribution of the cracks of rock samples with different joint spacings $\left(30^{\circ}\right)$
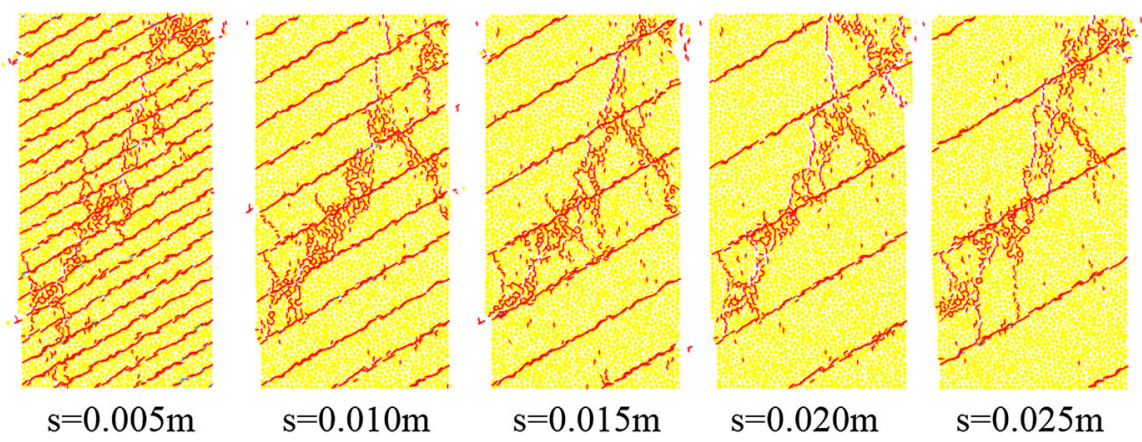

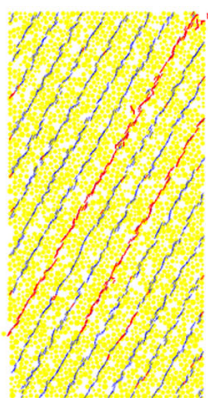

$\mathrm{s}=0.005 \mathrm{~m}$
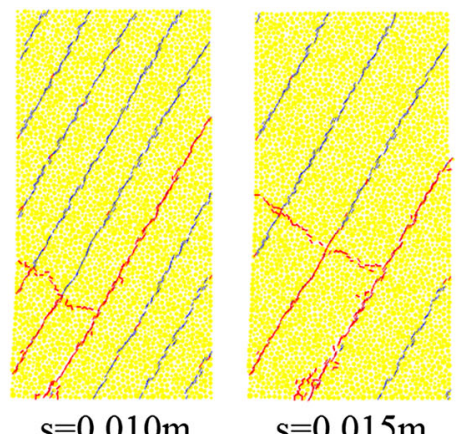

$\mathrm{s}=0.015 \mathrm{~m}$

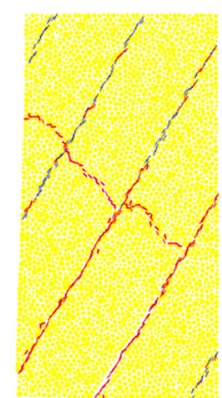

$\mathrm{s}=0.020 \mathrm{~m}$

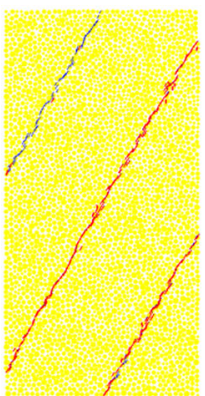

$\mathrm{S}=0.025 \mathrm{~m}$
Fig. 14 Distribution of the cracks of rock samples with different joint spacings $\left(90^{\circ}\right)$
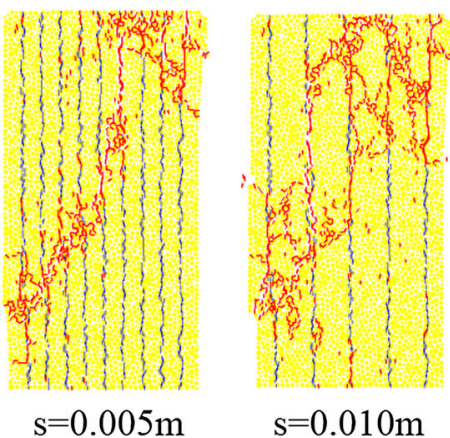
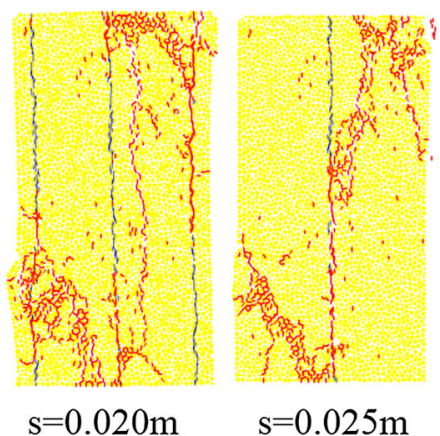

$\mathrm{s}=0.025 \mathrm{~m}$ spacing are in accordance with a quadratic polynomial function, and the elastic modulus of the rock mass with different joint inclinations increases with increasing joint spacing. The maximum difference ratio of compressive strength and elastic modulus is 1.36 and 1.59 , respectively under different joint spacings. In addition, Figs. 15 and 16 show that the normalized compressive strength is minimum under a joint inclination of $60^{\circ}$ but maximum under a joint inclination of $90^{\circ}$.

\section{Conclusion}

In this paper, the characteristics of rock mass anisotropy and its impact on strength and deformation has been evaluated using particulate DEM. Based on different arrangements of dip angle and spacing of a set of joints, a series of numerical experiments of uniaxial compression has been completed to study the effect of the joint plane angle and spacing on the rock mass deformation and strength. 
Fig. 15 Variation of the normalized compressive strength with the normalized joint spacing

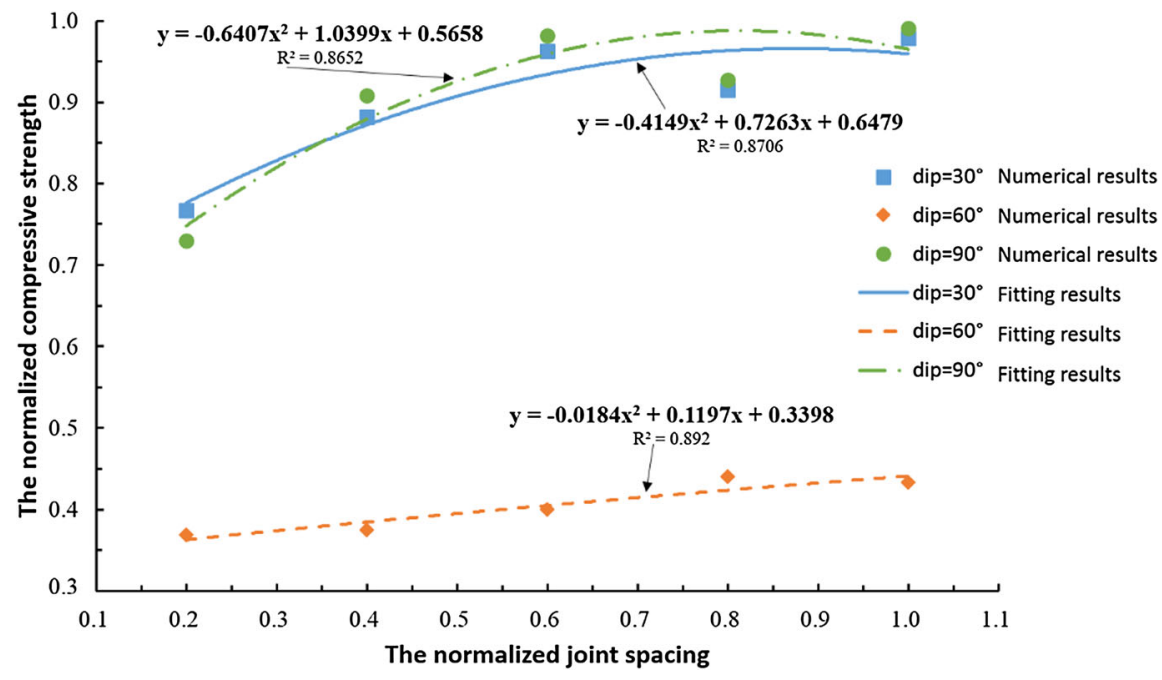

Fig. 16 Variation of the normalized elastic modulus with the normalized joint spacing

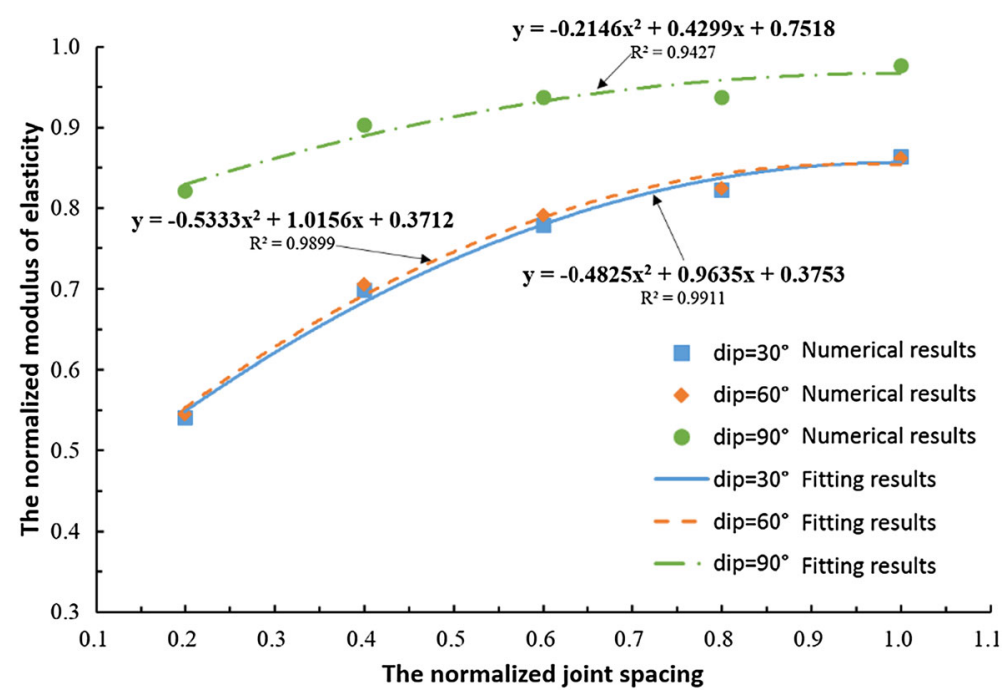

According to the complete stress strain curve recovered from the different numerical experiments of uniaxial compression for different joint dip angles, the distribution features of the micro-cracks, the number of micro-cracks and the rose diagram of the micro-cracks have all been analyzed. The resulting failure mode of jointed rock masses is found to be divided into three categories: splitting mode, sliding mode, and mixed mode. The strength and deformation of the rock mass presents an approximately " $U$ " shaped curve against dip angle. The maximum value of the strength and deformation parameters of the rock mass is obtained when the dip of the fractures is $0^{\circ}$ or $90^{\circ}$; the minimum value is obtained when the angle is $40^{\circ}-50^{\circ}$. The ratio of the maximum and minimum values of the compressive strength and the elastic modulus are 1.34 and 2.53 , respectively.

In view of the three types of failure modes, the dip angles of $30^{\circ}, 60^{\circ}$ and $90^{\circ}$, and five different joint spacings for each set are selected for uniaxial compression tests. The results show that the characteristics of the distribution of cracks are greatly influenced by the joint surface inclination, whereas different joint spacings have little influence. The largest difference ratio of the UCS and the elastic modulus are 1.36 and 1.59 , respectively, in the condition of different joint spacings and the same dip angle. 
Acknowledgments This study was funded by the National Natural Science Foundation of China (NSFC) under the Contract Nos. 51428902 and 51304237 , by State Key Laboratory of Coal Resources and Safe Mining under the Contract No. SKLCRSM14KFB06.

\section{References}

Amadei B (1982) The influence of rock anisotropy on measurement of stresses in situ $\mathrm{PhD}$ thesis. University of California Berkeley

Chiu C, Wang T, Weng M, Huang T (2013) Modeling the anisotropic behavior of jointed rock mass using a modified smooth-joint model. Int J Rock Mech Min Sci 62:14-22

Cho N, Martin CD, Sego DC (2007) A clumped particle model for rock. Int J Rock Mech Min Sci 44(7):997-1010

Cho JW, Kim H, Jeon S, Min KB (2012) Deformation and strength anisotropy of Asan gneiss, Boryeong shale, and Yeoncheon schist. Int $\mathrm{J}$ Rock Mech Min Sci 50(12):158-169

Chu WJ, Zhang CS, Hou J (2013) A particle-based model for studying anisotropic strength and deformation of schist. In: Proceedings of the 3rd ISRM SINOROCK symposium. Shanghai, China, p 593-596

Cundall PA, Strack ODL (1979) A discrete numerical model for granular assemblies. Géotechnique 29:47-65

Duan K, Kwok CY (2015) Discrete element modeling of anisotropic rock under Brazilian test conditions. Int J Rock Mech Min Sci 78:46-56

Everitt RA, Lajtai EZ (2004) The influence of rock fabric on excavation damage in the Lac du Bonnett granite. Int J Rock Mech Min Sci 41(8):1277-1303

Fakhimi A, Carvalho F, Ishida T, Labuz JF (2002) Simulation of failure around a circular opening in rock. Int J Rock Mech Min Sci 39(4):507-515

Itasca Consulting Group, Inc. (2010) PFC2D-Particle Flow Code in two dimensions, version 4.0 user's manual. ICG, Minneapolis
Hoek E (2007) Practical rock engineering. http://www. rocscience.com/education/hoeks_corner/

Jaeger JC (1972) Rock mechanics and engineering. Cambridge University Press, Great Britain

Jaeger JC, Cook NGW, Zimmerman RW (2007) Fundamentals of rock mechanics, 4th edn. Hoboken, Wiley

Kulatilake PHSW, Malama B, Wang J (2001) Physical and particle flow modeling of jointed rock block behavior under uniaxial loading. Int $\mathrm{J}$ Rock Mech Min Sci 38(5):641-657

Li Y, Yang C, Daemen JJK, Yin X, Chen F (2009) A new Cosserat-like constitutive model for bedded salt rocks. Int $\mathrm{J}$ Numer Anal Methods Geomech 33(15):1691-1720

Mas Ivars D et al (2011) The synthetic rock mass approach for jointed rock mass modelling. Int $\mathrm{J}$ Rock Mech Min Sci 48(2):219-244

Park B, Min K (2015) Bonded-particle discrete element modeling of mechanical behavior of transversely isotropic rock. Int J Rock Mech Min Sci 76:243-255

Wang T, Huang T (2009) A constitutive model for the deformation of a rock mass containing sets of ubiquitous joints. Int J Rock Mech Min Sci 46(3):521-530

Wang Y, Tonon F (2009) Modeling Lac du Bonnet granite using a discrete element model. Int J Rock Mech Min Sci 46(7):1124-1135

Wasantha PLP, Ranjith PG, Zhang QB, Xu T (2015) Do joint geometrical properties influence the fracturing behaviour of jointed rock? An investigation through joint orientation. Geomech Geophys Geoenergy Georesour 1(1-2):3-14

Yang ZY, Chen JM, Huang TH (1998) Effect of joint sets on the strength and deformation of rock mass models. Int J Rock Mech Min Sci 35(1):75-84

Zhang Q, Zhu H, Zhang L, Ding X (2011) Study of scale effect on intact rock strength using particle flow modeling. Int $\mathbf{J}$ Rock Mech Min Sci 48(8):1320-1328 